\title{
Outcome of combined surgical and intralesional steroids injection therapy for management of ear keloid Bajracharya A $\mathrm{D}$
}

Amir Bajracharya, Department of Plastic Surgery, Kathmandu Medical College Teaching Hospital, Sinamangal, Kathmandu, Nepal.

\begin{abstract}
Background: Ear keloid is one of the challenging problems that affect people of different races with substantial aesthetic outcomes. Various types of treatment modalities, including intralesional corticosteroid injection are advocated to lower recurrence following excision.

Objectives: To investigate the efficacy of a combined excision and postoperative intralesional triamcinolone acetonide (TA) injection for treating ear keloid patients.

Methodology: This was a descriptive study done to observe the outcome of combined approach of surgical and intralesional steroids injection therapy for ear keloids. Age, sex, site, size, duration, recurrence, and aesthetic outcome were evaluated.

Results: A total of 18 patients representing 19 ear keloids, with one case having bilateral and 3 pediatric cases were included from February 2018 to January 2019.The mean age was 22 years with female to male ratio of 5:1, site were left sided $9(50 \%)$, right $8(44 \%)$ and $1(6 \%)$ bilateral. About $10(53 \%)$ cases were at helix, and 9(47\%) at ear lobule. Mean length of ear keloid was $1.53 \mathrm{~cm}$ with range of $0.5-3 \mathrm{~cm}$ and mean breath $1.39 \mathrm{~cm}$ with the range of $0.5-2.5 \mathrm{~cm}$. The mean duration of ear keloid was 9.47 months. 2 (11\%) cases showed a history of recurrence. Injection triamcinolone acetonide hypersensitivity was noted by $1(5.3 \%)$ patient. Evaluation for all patients with aesthetic outcome was mean \pm standard deviation (4.38 \pm 1.025$)$.

Conclusion: Management of ear keloid using the combination of surgical excision and intra-lesional steroids injection therapy can be a good alternative option with low recurrence rate.
\end{abstract}

Key words: Ear keloid; Intra-lesional steroids, Surgical excision.

\section{INTRODUCTION}

$T$ eloid is an adverse scar that grows beyond the borders of previous scar, characterized by excessive

\section{Access this article online}

Website: www.jkmc.com.np

DOI: https://doi.org/10.3126/jkmc.v9i1.33544

\section{HOW TO CITE}

Bajracharya A. Outcome of combined surgical and intralesional steroids injection therapy for management of ear keloid. J Kathmandu Med Coll. 2020;9(1):43-48.

\section{Address for correspondence}

\section{Dr. Amir Bajracharya}

Assistant Professor, Department of Plastic Surgery

Kathmandu Medical College Teaching Hospital

Sinamangal, Kathmandu, Nepal

E-mail: amirbajracharya@hotmail.com

Copyright @ 2020 Journal of Kathmandu Medical College (JKMC)

ISSN: 2019-1785 (Print), 2091-1793 (Online)

(i) (S) This work is licensed under a Creative Commons Attribution-Non Commercial 4.0 International License. deposition of collagen in the dermis during the wound healing process ${ }^{1}$. Keloid and hypertrophic scar are the adverse scars which are usually difficult to manage. The term keloid comes from the Greek word 'Chele" meaning crab claw like appearance ${ }^{2,3}$. The most common affected areas are the anterior chest, shoulders, flexor surfaces area of the extremities, and the ears ${ }^{4}$. Keloid formation is about 15 times higher in black people than in whites ${ }^{5}$. Surgical excision alone leads to a high recurrence rate between $50-100 \%$, therefore combination of surgical excision with steroids therapy, radiotherapy, mechanical compression, silicon occlusive dressings, cryosurgery, and bleomycin tattooing etc are being used as the treatment modalities for management of keloid ${ }^{6,7,8}$.

Corticosteroids injections are used to treat keloid. The mechanism of action of corticosteroids are primarily due to their suppressive effects ${ }^{9}$ on the wound inflammation and secondarily due to reduction in the synthesis of collagen and glycosaminoglycans and inhibition of fibroblast growth ${ }^{10}$, and thirdly due to enhanced 
collagen and fibroblast degeneration ${ }^{11}$. The most used corticosteroid is triamcinolone acetonide with a dose of $10-40 \mathrm{mg} / \mathrm{ml}$, and it is given intralesionally in an interval of 3-6 weeks ${ }^{12}$. The side effects of the drugs include hypo or hyper-pigmentation, skin atrophy, telangiectasia and ulceration $^{13}$. In this study we investigated the efficacy of a combined treatment protocol using excision with postoperative intralesional triamcinolone acetonide (TA) injection for treating ear keloid patients.

\section{METHODOLOGY}

This descriptive study was carried out in the Department of Plastic Surgery, Kathmandu Medical College Teaching Hospital, Sinamangal, and Kathmandu from February 2018 to January 2019. Institutional ethical committee approval was taken before conducting the study with informed patient consent. The objective of the study was to assess the outcome of a combined method of surgical and intra-lesional steroid therapy for management of ear keloid. We included all patients with bilateral or unilateral ear keloids who presented to the plastic surgery department of Kathmandu medical college, Sinamangal. Exclusion criteria included patients with a history of hypersensitivity to steroid injection. Age, sex, site, size and duration of ear keloid, recurrence rate were analyzed. Standardized photographs were taken preoperatively, immediately after the surgery, and during the follow up. The patients were recalled 2 weeks after surgery for suture removal and for injection of $1^{\text {st }}$ dose of intralesional steroid therapy. Further the patients were recalled for consecutive 3 weeks for $2^{\text {nd }}$ to $5^{\text {th }}$ dose of intralesional steroid therapy. The patients were followed up post therapy every 3 months up and the follow up period was carried out till December 2019. The aesthetic aspects and outcome were evaluated by the patient after the complete treatment using the following grading scale: 1 , poor; 2 , fair; 3 , good; 4 , very good; and 5, excellent and the scaling was done by the patient. Frequency analysis was done for the entire cases recorded using Statistical Package for the Social Sciences software version 20, Chicago, IL, USA.

\section{SURGICAL PROCEDURE}

Based on the pre-operative evaluation of the patient, local anesthesia of $1 \%$ diluted lignocaine with hypodermic needle 26 gauge was used, ear keloid was excised or extirpated; depending upon the defect and local flaps was used to cover the post-excisional defects decided by the operating surgeon. Post operatively the patients were advised to take one week of antibiotics therapy of oral capsule cloxacillin as per patient body weight with oral tablet paracetamol as per requirement for pain management.

\section{RESULTS}

A total of 18 patients representing 19 ear keloids were recorded within the duration of one year from February 2018 to January 2019. Among these, 3 pediatric patients with small ear keloids were managed non-operatively and were closely monitored by regular follow-up. The 15 patients representing 16 ear keloids were included for surgical and intra-lesional steroids injection therapy. Demographic profile for all patients presented within a one-year period with the keloid data is given in Table 1. The mean age was 22 years with range from 3- 38 years. Most of them were female with female to male ratio of 5:1. In our study $2(11 \%)$ patients gave histories of recurrence and $1(5.3 \%)$ patients showed postoperative hypersensitivity to the first dose of injection triamcinolone acetonide, which was then discontinued and was managed accordingly.

Evaluation for all patients with overall aesthetic outcome was done by the patient themselves after complete treatment, by using a grading scale of: 1 , poor; 2 , fair; 3 , good; 4, very good; and 5, excellent with mean \pm standard deviation $4.38 \pm 1.025$.

Table 1: Demographic and Keloid data of the patients

\begin{tabular}{lc}
\multicolumn{1}{c}{ Variables } & Results \\
Number of Patients & 18 \\
Age in years & 22 \\
Mean & $3-38$ \\
Range & \\
Sex & $3(17 \%)$ \\
Male & $15(83 \%)$ \\
Female & \\
Keloid (n=19) & $9(50 \%) / 8(44 \%) / 1(6 \%)$ \\
Side (left/ right/bilateral) & $10(53 \%) / 9(47 \%)$ \\
Site (helix / ear lobule) & $9.47 \mathrm{mths}(3-24 \mathrm{mths})$ \\
Duration (range) & $1.53 \mathrm{~cm}(0.5-3 \mathrm{~cm})$ \\
Length (range) & $1.39 \mathrm{~cm}(0.5-2.5 \mathrm{~cm})$ \\
Breadth (range) &
\end{tabular}



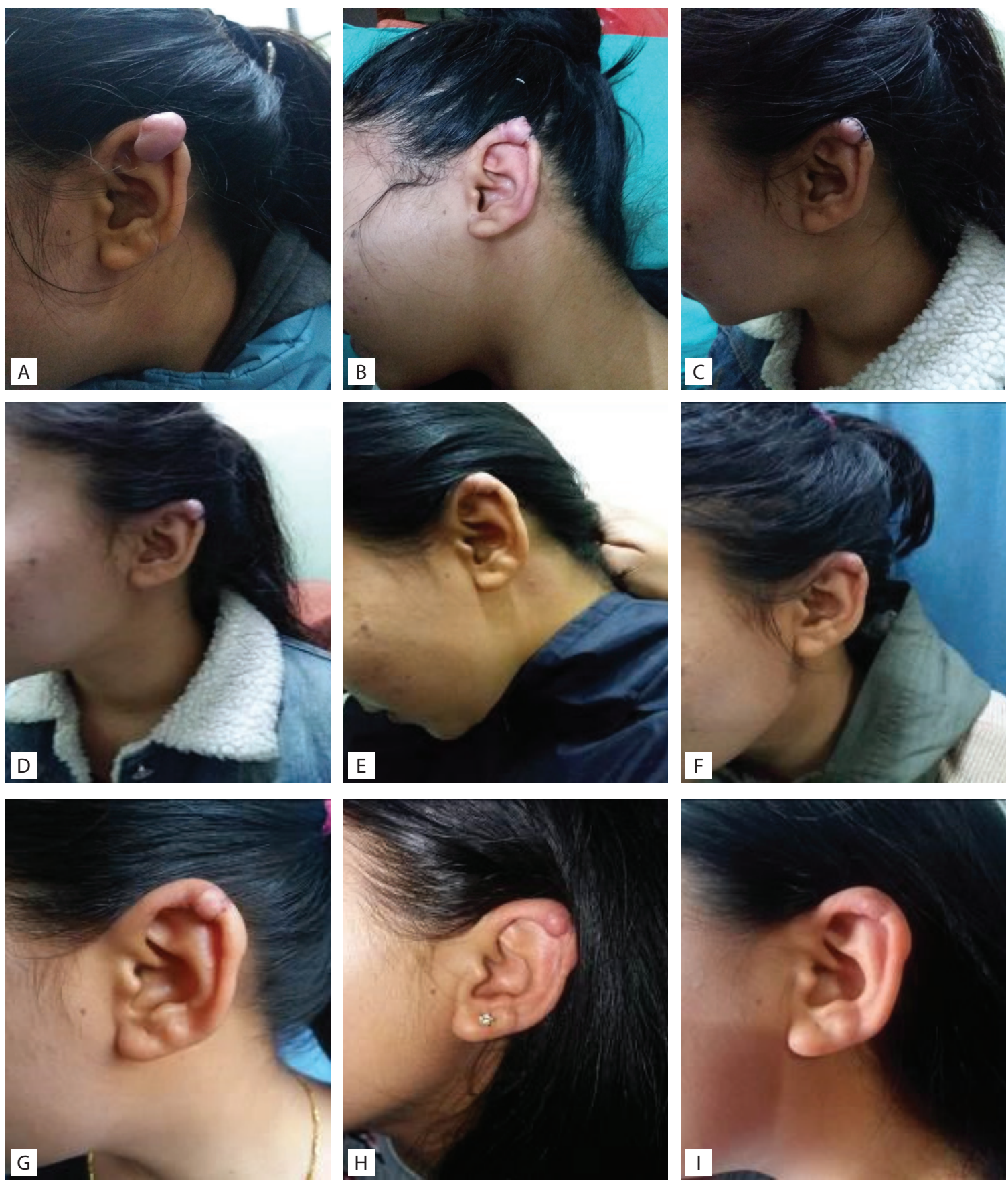

Figure 1: 26 years female with recurrent ear keloid of left helix (A: pre-operative, B: post-operative, C: 1 week post-operative, D: during suture removing time and first dose of injection triamcinolone, E: after second dose, F: after third dose, G: after fourth dose, H: after fifth dose, I: follow up after six months). 

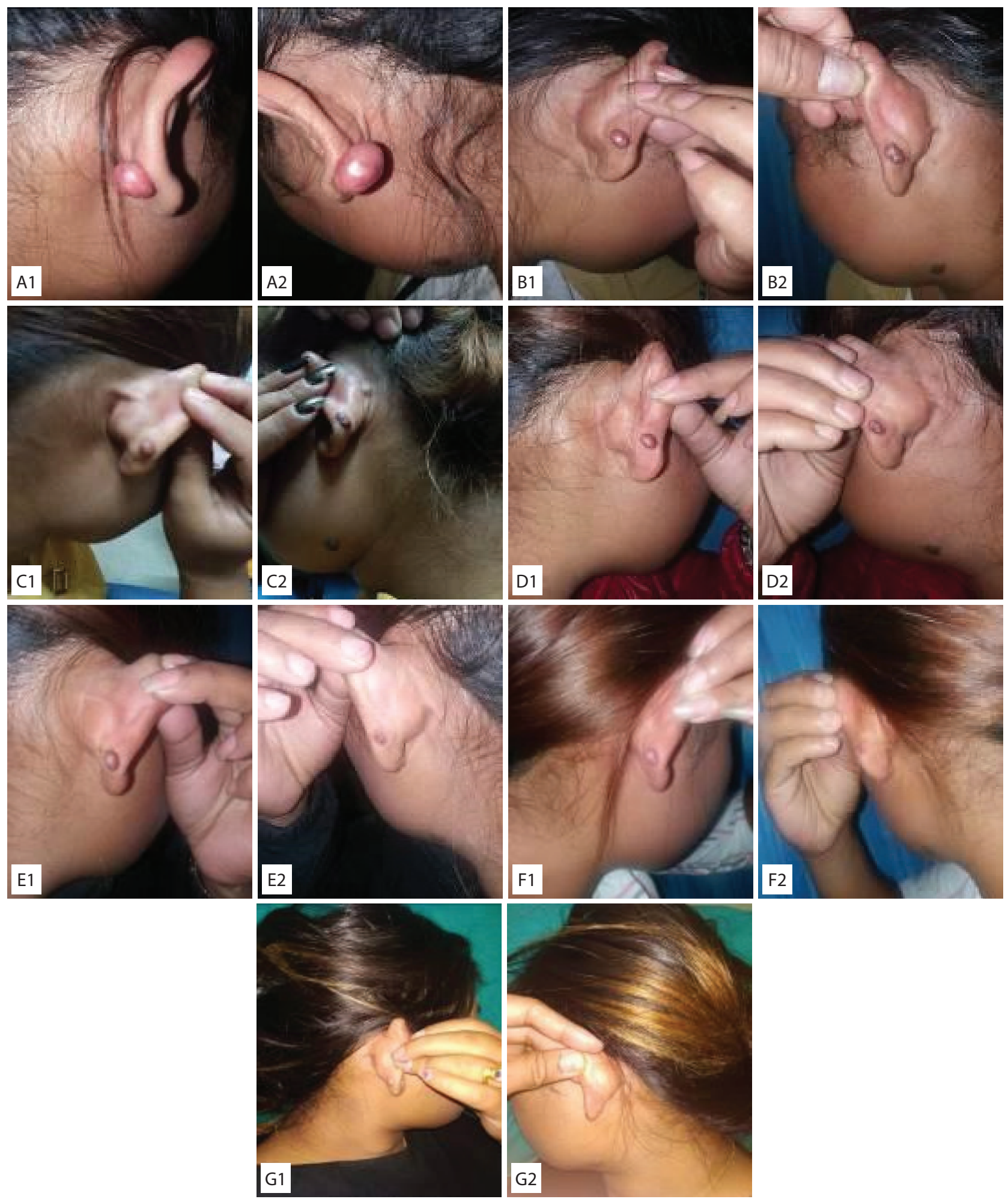

Figure 2: 30years female with bilateral posterior ear lobule ear keloid (A1\&A2: Right and left Pre-operative, B1 \&B2: Right and left post operative after 1 st dose of injection triamcinolone, C1 \&C2: Right and left after second dose, D1 \&D2: Right and Left after third dose, E1\&E2: Right and left after fourth dose, F1 \& F2: Right and left after fifth dose, G1\&G2: Right and left follow up after six months) 

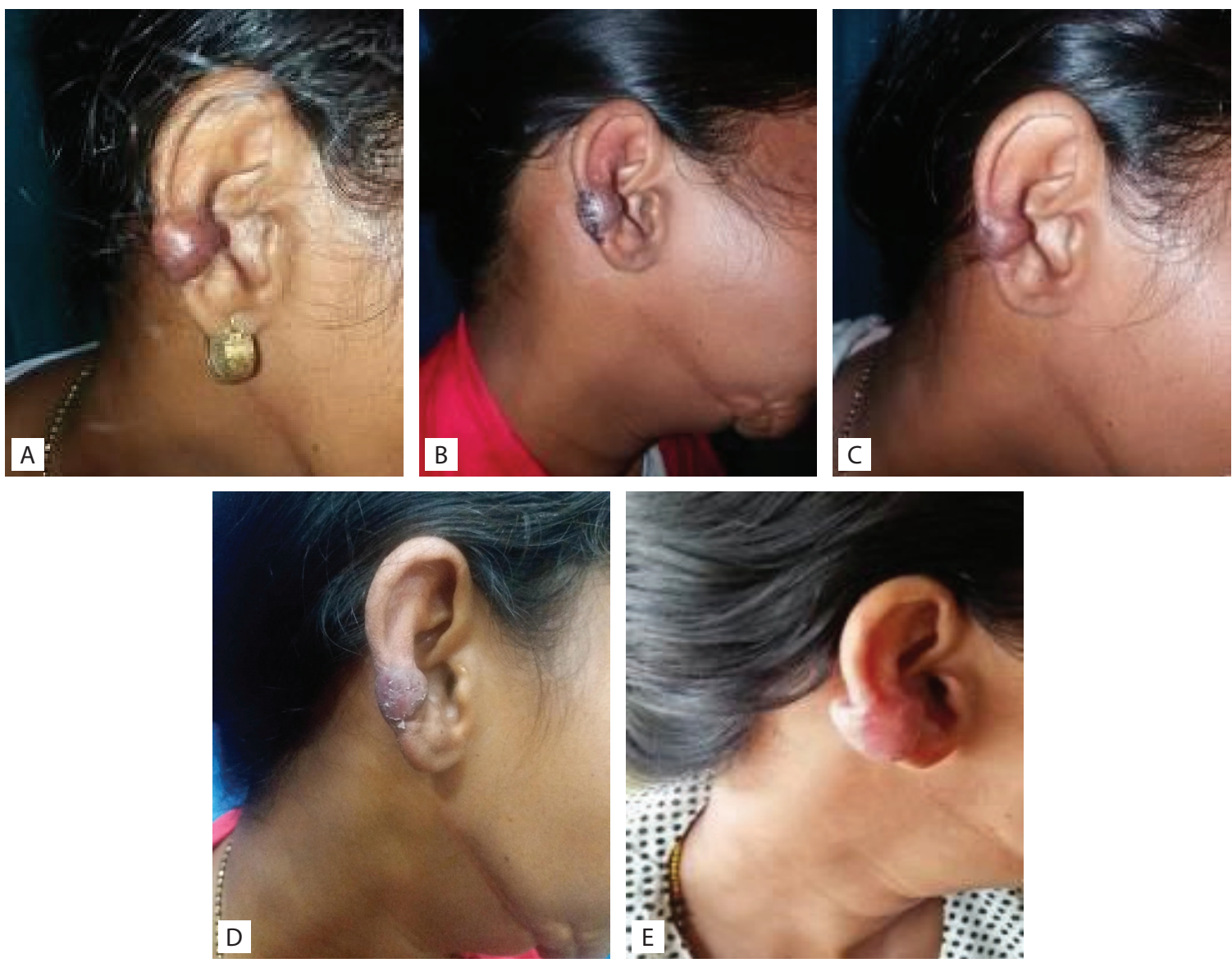

Figure 3: 38years female with right helical ear keloid (A: Pre-operative, B: post operative 2 weeks at the time of suture removal, she developed hypersensitivity to injection triamcinolone: C: Follow up after 3 weeks post-operative, D: followup after 6 weeks, E: 6 months follow-up.

\section{DISCUSSION}

This descriptive cross-sectional study was done to assess the outcome of a combined method of surgical and intra-lesional steroid therapy for management of ear keloid. Although there is a lot of information about histomorphological structure of keloids in current literature, there is no guideline regarding the treatment. There are various treatment methods described, such as surgical excision, intralesional injection of corticosteroids, compressive therapy, radiotherapy, laser therapy, cryosurgery, therapy with antitumor or immunosuppressive agents and combinations of these methods. The reported success rate is variable. This is why we chose combined treatment modalities to get the acceptable results. Surgical excision alone leads to a high recurrence rate, between $50-100 \%$ 6,7,8. Therefore it is rarely used as single therapy.

Surgical removal of excessivescartissuereturnsthewound to the initial state and further postoperative scarring can be reduced by supportive therapies like intralesional corticosteroid injections, radiotherapy, pressure therapy, and immunomodulators. A retrospective cohort study found that multimodal therapy (excision followed by combination of steroid and radiation therapy or steroid and silicone gel therapy) showed less recurrence than post excisional steroid alone ${ }^{8}$.

Corticosteroids can be used as first line therapy, with good response rates from 50 to $100 \%$ and recurrence rates between $9 \%$ and $50 \%{ }^{12}$. Side effects which include hypo or hyper-pigmentation, telangiectasia, skin atrophy and pain upon injection can be encountered in up to $63 \%$ of patients ${ }^{13}$. Injection Triamcinolone acetonide can be used in a concentration of $40 \mathrm{mg} / \mathrm{mL}$, at monthly intervals for up to 6 months ${ }^{14}$. When used in conjunction with surgery, local steroid therapy is initiated before surgery and continued postoperatively, monthly, for at least 3 months ${ }^{13,15}$. Rosen et al ${ }^{16}$ treated ear keloids with excision and intraoperative and postoperative 
injection of steroids and reported a recurrence rate of $23 \%$. Shons et al. ${ }^{17}$ evaluated 31 earlobe keloids in 20 patients, they found after surgical excision of the scar and adjunctive therapy using three postoperative injections of triamcinolone; in a follow-up period of 12 to 62 months, only 1 recurred which was similar to our data which also showed recurrence in 1 (5.3\%) case due to hypersensitivity to injection triamcinolone acetonide. The patient was admitted for overnight observation, as the patient developed skin rashes and injection antihistamine was given on the next day she was discharged on satisfactory conditions.

The cases in this study are from a single institution and has single surgeon experience so it may have some limitations. It was just an observational study, comparative or RCT would have been better. No blinding

\section{REFERENCES}

1. Murray JC. Scars and keloids. Dermatol Clin 1993;11:697-708. [PubMed]

2. Jackson IT, Bhageshpur R, DiNick V, Khan A, Bhaloo $S$. Investigation of recurrence rates among earlobe keloids utilizing various postoperative therapeutic modalities. Eur J Plast Surg.2001;24(2):88-95. [Full Text]

3. Leventhal D, Furr M, Reiter D. Treatment of keloids and hypertrophic scars. A meta-analysis and review of the literature. Arch Facial Plast Surg. 2006;8(6);362-8. [PubMed]

4. Burd A, Huang L. Hypertrophic response and keloid diathesis; two very different forms of scar. Plas Reconstr Surg 2005;116:150-7. [PubMed]

5. Moshref SS, Mufti ST. Keloid and Hypertrophic Scars: ComparativeHistologicaland Immunohistochemical Study. Med Sci,2010;17:3-22. [DOI]

6. Berman B, Perez OA, Konda S, et al. A review of the biologic effects, clinical efficacy, and safety of silicone elastomer sheeting for hypertrophic and keloid scar treatment and management. Dermatol Surg 2007;33(11):1291-1303. [PubMed]

7. Nast A, Eming S, Fluhr J, et al. German S2k guidelines for the therapy of pathological scars (hypertrophic scars and keloids). J Dtsch Dermatol Ges, 2012; 10(10):747-762. [PubMed]

8. Lindsey WH, Davis PT. Facial keloids. A 15-year experience. Arch Otolaryngol Head Neck Surg, 1997; 123:397-400. [PubMed] was done so observer bias was there, multicenter study with double blind would have been better.

\section{CONCLUSION}

Outcome of combined Surgical and intra-lesional steroids injection therapy can be a suitable modality for management of Ear Keloid. The treatment method gives good aesthetic outcome and low recurrence rate.

\section{ACKNOWLEDGEMENTS}

I would like to acknowledge Kathmandu Medical College Teaching Hospital, Late Prof. Shambhu Nath, Dr. Sandesh Maskey, Dr Abhinav Vaidya, Dr. Naresh Manandhar and Dr. Dipshikha Bajracharya for their valuable support.

Conflict of interest: None

Source(s) of support: None

9. Reish RG, Eriksson E. Scar treatments: preclinical and clinical studies. J Am Coll Surg, 2008; 206(4):719730. [Full Text]

10. Cruz NI, Korchin L. Inhibition of human keloid fibroblast growth by isotretinoin and triamcinolone acetonide in vitro. Ann Plast Surg, 1994; 33(4):401405. [PubMed]

11. Boyadjiev C, Popchristova E, Mazgalova J. Histomorphologic changes in keloids treated with Kenacort. J Trauma, 1995; 38(2):299-302. [PubMed]

12. Niessen FB, Spauwen PH, Schalkwijk J, Kon M. On the nature of hypertrophic scars and keloids: a review. Plast Reconstr Surg, 1999; 104:1435-1458. [Full Text]

13. Brown LA Jr, Pierce HE. Keloids: scar revision. J Dermatol Surg Oncol. 1986; 12(1):51-56. [Full Text]

14. Brissett $A E$, Sherris DA. Scar contractures, hypertrophic scars and keloids. Facial Plast Surg, 2001; 17:263-272. [Full Text]

15. Jung JY, Roh MR, Kwon YS, Chung KY. Surgery and perioperative intralesional corticosteroid injection for treating earlobe keloids: a Korean experience. Ann Dermatol, 2009; 21(3):221-225. [PubMed]

16. Rosen DJ, Patel MK, Freeman K, Weiss PR. A primary protocol for the management of ear keloids: results of excision combined with intraoperative and postoperative steroid injections. Plast Reconstr Surg, 2007; 120:1395-1400. [PubMed]

17. Shons $A R$, Press $B H$. The treatment of earlobe keloids by surgical excision and postoperative triamcinolone injection. Ann Plast Surg, 1983; 10:480-482. [Full Text] 